\title{
Chapter X. Studying Complex Care Transitions from a Quantitative Perspective
}

\author{
Line Kildal Bragstad, 1.k.bragstad@medisin.uio.no \\ Christina Foss, i.c.foss@ medisin.uio.no
}

\section{Introduction}

This chapter focuses on how transitional care can be studied from a quantitative perspective. By a quantitative perspective we mean use of methods for gathering data that may be expressed numerically, and that are amenable to mathematical analysis that enables the researcher to describe variables and to examine relationships between them using statistical methods. Using a quantitative design in the field of transitional care can be performed by use of pre-existing 'routine' service data, like for instance regional or national register data or hospital discharge summaries combined with readmission rates, depending on the subject and research questions that are to be investigated. Another way, perhaps more often used, is by gathering data through using a pre-existing questionnaire or by developing one within the project. Drawing on Polit \& Beck (2017) we use the term survey research in describing approaches using questionnaires.

Quantitative data are useful for monitoring and improving the quality of transitional care. For example, quantitative data can aggregate patient or professional experiences to identify failures, weak points or clogs in the transition process. Also, studies can be repeated, and thus be useful in monitoring change over time or to measure effects of an intervention. They can provide a knowledge basis for comparison between routines in different hospital wards or for comparison between comparable wards at different hospitals ${ }^{1}$. Quantifiable data can also be used to compare perceptions of different groups, like for instance between patients and professionals. In short, although surveys, like all research designs have limitations, they are important research tools that have a potential to contribute to knowledge that can help reduce patients' sufferings and stress, reduce adverse events and costly readmissions.

To frame and ground the discussions in this chapter we will draw on experiences from the research study "Transitions from hospital to community care" (referred to as the "Transition study" after this), where we used a descriptive cross-sectional design with a consecutive sampling procedure

\footnotetext{
${ }^{1}$ In Norway every hospital uses the same questionnaire to measure patient experiences and this is published on a web site where patient satisfaction is used as one of several quality indicators patients can use for selecting hospitals.
} 
collecting data from elderly patients and their next of kin (Bragstad et al., 2014, Bragstad et al., 2012, Foss and Hofoss, 2011). Surveying transitional care can be based on exploring the experiences of different stakeholders; professionals, patients and next of kin. In the Transitions study, we developed two versions of an instrument; The Discharge of Elderly Questionnaire (DEQ), one targeting the experiences of elderly patients and the other the experiences of their next of kin. The next of kin questionnaire was developed with minor adjustments compared to the patient questionnaire. The DEQ was designed to elicit data concerning the subjects' experiences regarding the discharge and the management of their health problems after discharge.

The aim of this chapter is to highlight methodological challenges in studying transitional care from a quantitative perspective. It is impossible to justly present the wide range of methodological approaches within a quantitative perspective; thus, experiences from the Transitions study inform this chapter, and the examples used will mainly stem from approaching the field of study with a cross-sectional survey design.

\section{Studying transition step-by-step}

The different steps in the planning and implementation of a survey study can be described in different ways and through use of different headings. In this chapter we will present the theme, surveying transitional care, under the following headings;

- Designing and planning the study

- Development of the instruments

- Conducting survey research on transitional care

Having stated this, it is important to also remind the reader that research processes are very rarely linear (even in quantitative designs). In reality we moved back and forth, for instance between different specifications, construction of the questionnaire and plans for recruitment of respondents. Although these processes are not linear, that does not mean you are exempt from the requirement to have a well thought out plan.

\section{Designing and planning the study}

\section{Specifying the purpose and population}

Planning the study often means going back and forth to ensure correspondence between the purpose of the study and the target population for data collection. Transitional care is, as shown in Chapter 1 in this book, a complex concept and includes a range of different elements, systems, levels and professions. Consequently, transitional care is difficult (impossible) to capture in its full breadth, 
and it is vital to be as specific as possible. In our case the purpose was to gain information of elderly patients' experiences of the transition process between the two levels of care; hospital care to municipal care. To specify further we narrowed our scope by determining that our target population was old persons (80+) as this population is identified as especially vulnerable during transitions. We wanted to include patients who were discharged from hospital to municipal care with home care or to nursing home care ${ }^{2}$. Experience from the field indicated that this is a group of patients that often rely on the assistance and support from their next of kin, and thus we wanted to include their experiences. As a way of recruiting next of kin instead of extracting information from the patient records, we let the patient specify the person they viewed as their next of kin.

\section{Specifying when in the discharge process we can get the information we need}

Another issue in conducting research is about when to collect data - in what phase of the transition process should the survey be administered? We made a (broad) decision that we would gather data after patients were discharged from hospital back to their home or nursing home. The time of data collection will be further discussed in the chapter discussing how to conduct research on transitional care.

\section{Development of the instruments}

To collect valid information on patients' and next of kin's experiences of the transition from hospital to municipal care, several considerations needed to influence the construction of the questionnaire. As main categories we can separate between the "what" and the "how", what questions to ask and how to ask (including categories to answer) them.

\section{The what; selecting the substantial content of the questionnaire}

Basic in validity is to obtain a good construct validity, hence, we needed to ensure that the questionnaire measured what we intended. The standard recipe found in many method books for developing good questions is that you must first identify the theoretical basis for your survey, and then formulate specific hypotheses to test. This is a recipe for a theory-governed question development or a "top-down" strategy using a deductive approach (Kearney, 2016b, Kearney, 2016a). However, in many surveys it can be difficult to spot any theoretical basis for testing hypotheses. In our study the aim was to identifying problem areas rather than to test hypotheses, and thus the questionnaires had a primarily practical purpose. We did not have one distinct

\footnotetext{
${ }^{2}$ Patients discharged from hospital to home without home care is of course also an interesting group, but as the focus of the study was on transitions between different levels of care, this group was outside the focus of our study.
} 
hypothesis that we wanted to test, but based on earlier research we did assume that there would be some distinct areas that would be of significance. One theoretically based assumption that we made was that levels of participation in decision making would influence the patients' and next of kin's experiences during the transition process.

A basic requirement in survey design is to be knowledgeable of the area you wish to research. The development of a questionnaire can be informed by focus groups and interviews with patients or with professionals (Coleman, 2003). In our study, we had important input from a qualitative study collecting data of elderly patients' experiences with discharge from hospital to home (Foss, 2011). The process of developing a questionnaire was followed by searching the literature on qualitative studies reporting elderly patients' own accounts of their discharge process with the aim to identify substantive areas that was deemed important to old patients themselves. From the existing literature we were able to identify four main areas, or dimensions described to be important in the discharge process;

- Time of discharge,

- Elements in the community health care,

- Practical matters

- Issues related to medication and other treatment after discharge.

These dimensions formed the basis of development of questions in the development of the survey.

\section{The how; selecting the measures of the questionnaire}

Selection of measures appropriate for the specific patient population and service setting was necessary. Based on review of the literature and of existing questionnaires (Foss and Askautrud, 2010), we wanted data on how patients and next of kin experience participation in the discharge process. How is it possible to measure participation? Operationalization of the concept we want to investigate is often related to the theoretical understanding of the issue under investigation. The question of how to operationalize participation was inspired by Thompson (2007), and using this taxonomy on levels of participation made it possible for us to determine what level of participation that was measured in the existing instruments. Thompson identifies five levels of patient involvement: (0) Non-involvement - where the patients are just passive recipients of care and treatment; (1) Information seeking/information receptive - a stage that is not seen as involved per se, but rather a requirement for being able to take part in decision-making about their own care and treatment; (2) Information dialogue - incorporating the exchange of information between the patient 
and clinicians; (3) Shared decision-making - where patients and clinicians cooperate to find the best solutions and the patients' experiences that their opinions are taken in account when decisions are made; (4) Autonomous decision-making - a level that indicates independent decision-making for the patients, without confronting the clinicians. We did not use the two most extreme levels (0 and 4). By use of levels 1, 2, and 3 for instance the questions related to practical help after discharge below were graded on a four-point scale from agreement to disagreement:

1. I received information on what help I would receive after discharge.

2. I got the opportunity to tell the staff what I myself considered important in order to manage after discharge

3. We co-operated on how to solve different practical issues

The development of questions is influenced by the desired or feasible size of the overall questionnaire, and the number of questions that the respondent has to answer tends to correspond with the tendency to actually finish the questionnaire. In our case, we needed to take into account that the respondents were old persons that had just been hospitalized and thus were likely to tire easily. We tested the questionnaire in the research team and made an estimate of how long it would take to answer the questions (in our case an average of 40 minutes) $)^{3}$.

A rule-of-thumb is to be precise (not general) and to avoid complex issues (part up questions). We also faced the typical challenge to pose questions that are unambiguous and sufficiently detailed to illuminate each dimension as good as possible. Initially, we had thorough discussions in the research team to remove ambiguities and confusions and in the process we went back and forth between the questions and what answers the questions were likely to trigger. For example, we found that (in our Norwegian language) one question could be understood both as asking "did you get to tell what was important for you" whereas some assumed that the same question meant "where you told what was important to you".

The design of the questionnaire is of great importance, and a good design makes the questionnaire easy to read and to orient in. To make the questions logical to the responders, the different parts or dimensions in the survey were organized and divided (as chapters in a book) with clear headings. The different dimensions were organized according to a natural association (for instance from admission to discharge). In the Transition-study, the two questionnaires were organized in four

\footnotetext{
${ }^{3}$ This was later tested with a sample from the study population as described under the piloting of the questionnaire.
} 
sections: "Demographic background", "Participation in the discharge process", "After discharge" and "Summary". In the "Demographic background" section patients were asked their demographic background and next of kin were asked about their relationship to the patient, the patient's hospital stay, and their own demographic background. The "Participation in the discharge process" section included questions designed to measure different aspects of patients' and next of kin's involvement and participation in the discharge process. Four questions were designed to measure the next of kin's perception of information practices at the hospital. In addition, four questions measured the next of kin's perceptions of how they were treated at the hospital. The "After discharge" section included patients' and next of kin's assessment of the first period after discharge, and questions on the amount of formal and informal care provided before admission and after discharge and on how the experienced the period after discharge. The "Summary" section included questions regarding patients' and next of kin' assessment of assistance received from and their trust in the health care services.

Fixed and given options have the advantage of providing information on how answers are categorized, and give information of the level of detail in the answers. If we for instance want to inquire on how an older person discharged from hospital to home with home-care lives, it is important not only to know if they live in a flat but also to know if the respondent lives in a flat on the fourth floor and if it is a building with or without an elevator. Fixed answers, however, place great demands on the construction of categories. Categories should be short and mutually exclusive, and they must cover all significant alternatives. If we want to use the alternatives "do not know" or "other", it is often wise to combine this with an open space and invite the respondent to elaborate on their specified answer. The "do not know" category should thus only be an option if this answer is regarded likely and natural; for instance the respondent is not likely to know when information were transferred from hospital to home care services, but may be more likely to know if they received information of the date of discharge prior to the date of discharge or not. In use of graded scales there is always a discussion of how many alternatives we need. If questions are to measure satisfaction the grading in even or odd numbers are decisive of the possibility to grade (or dichotomize) the answer as mostly positive or negative. The number of options in the grading of answers may increase the level of precision, but if there are too many the respondents may have problems grading. If possible, there should be a common logic in the ordering of alternatives, often placed in order of decreasing or increasing satisfaction, sufficiency, strain, or agreement. In our study, like in most surveys, a part of the questionnaire also entailed questions obtaining demographic data. An advice that we followed was to use already existing (and well tested) 
questions, after having acquired the necessary permissions from those that had developed and tested these questions.

\section{Piloting the questionnaire}

Face validity was not only developed through expert opinions among relevant groups like nurses and researchers in the field, but obviously also through the target population, i.e. the respondents. We performed pilot interviews to ensure that the questions were understandable and that they covered areas that the respondent felt were significant.

The term pilot study is used in different ways, but in the following we use the term to refer to the pre-testing or 'trying out' of a particular research instrument. Piloting a questionnaire is one way of letting the receiver or respondent co-author the development of the questionnaire. We looked at the survey as a form of two-way communication, where it was imperative to adjust formulations depending on the response from the respondent during piloting.

We did not have any elderly persons participating as partners in the project. Looking back, it would have been possible and probably also useful in the development of the questionnaire. User participation in research is increasingly used in contemporary research (Abma et al., 2009), but even in cases where users have been participating in development of the theme and dimensions of the survey, it may be wise to pilot the questionnaire. The importance of securing variety in respondents in the pilot will help assure that different understandings within different groups of the population promote a united understanding of questions posed, by the feedback of clarity and appropriateness and by investigating if important aspects are missing (Kearney, 2016b, Kearney, 2016a). In our study, we administered the questionnaire to pilot subjects in the same way as it were to be administered in the main study, and hence we did personal interviews with old patients (80+). We asked the subjects for feedback to identify ambiguities and difficult questions. To estimate the feasibility of the data gathering, we also made estimates of the time it would take to complete the interview. In our case we found that the estimate we had calculated during the planning phase corresponded with reality. The piloting was followed by a process of revision where we re-worded and/ or re-scaled any questions and answers that was unclear or not logical to the respondents.

Summarized, one may say that the first phase in the development of a questionnaire is to clarify what should be the raw material in question and then what the question structure should look like. The next element is to ensure that every single question and answer is formulated is a way that 
enables respondents to answer as intended. Finally, the instrument needs to be tested in a pilot study using respondents and data collection method that is comparable to the planned of survey.

\section{Conducting survey research on transitional care}

\section{Data collection}

As shown above, designing a research study with a quantitative approach entails making important decisions regarding not only choosing an appropriate instrument or indeed constructing an instrument and deciding what kinds of data to collect, but also in choosing how and when to collect data. These main features on how and when to collect the data are decided already in the design and planning of the study. There are a number of important decisions that will influence the success of the research study in reaching its goal of developing new knowledge on transitional care. In this section we will outline a few important methodological issues pertaining to data collection when studying transitional care from a quantitative perspective.

\section{Recruitment of respondents}

Identifying the appropriate study population is the first stage; the real challenge lies in successfully recruiting a large enough sample of the population into the study. In the Transitions study we relied on recruiting the patients through (publicly employed) community nurses responsible for the allocation of patients discharged from hospital to home care or nursing home. Although the nurses in the chosen municipalities agreed to notify the research team when they received notice of discharged patients that fit the study's inclusion criteria, in reality many forgot to do so in their hectic day-to-day clinical practice. As a result the recruitment process turned out to be long and troublesome for the research team, and we had to include many new municipalities to reach the number of respondents we needed. Looking back, we believe that we did not put enough effort into establishing a close enough contact with the "gatekeepers" in the municipalities to secure priority of recruiting patients to the study.

\section{Mode of data collection}

In choosing to approach the field of transitional care with a cross-sectional self-report survey design, there is a distinct flexibility in the options available regarding the actual mode of data collection; in-person interviews, telephone interviews, online questionnaire or mailed questionnaires. All of these approaches have their advantages and disadvantages, which may vary between different study populations and stakeholders. Often the decision is related to characteristics 
of the respondent, combined with an estimate of financial and personnel resources available in the research project. An online questionnaire may be appropriate and especially cost-effective in surveying health care personnel and healthy next of kin, however, this approach may be too demanding for frail elderly patients. In-person interviews may be more appropriate in surveying frail elderly patients even though it is very resource intensive, while telephone interviews may be a more cost-effective and sufficiently appropriate approach surveying next of kin. Importantly, most of these decisions reflect sound methodological choices to maintain the scientific quality of the study, but sometimes practical or pragmatic factors may also influence decision-making.

In the Transitions study, patients were interviewed in person and the next of kin by telephone. Inperson interviews were the method of choice due mainly to assumptions about the superiority of personal interviews as opposed to mail or online questionnaires in surveying the chosen population. Additionally, there is a unanimous perception among survey researchers that in-person interviews are advantageous in acquiring complete answers to questionnaires. It is easier to skip answering a question when completing a questionnaire per mail or online ${ }^{4}$ than in personal interviews. Making it impossible to skip a question may, however, discourage respondents from completing a questionnaire if they do not wish to answer certain (sensitive) questions of if the questionnaire is perceived as too long. The frailty of the patients due to their advanced age and recent hospitalization was thought to hamper their ability to complete self-administered questionnaires to be mailed or completed online. The issue of frailty or overall weakness needs to be addressed also when surveying transitional care in other patient populations; the transition process in itself is a time of vulnerability and possible threats to patient safety. We believed that an in-person interview would be less demanding and would positively influence the understanding of the questions and prevent missing answers in the Transition study. A possible disadvantage of in-person interviews is that the personal encounter may interfere with the strict consecutive sequence of the questions of the survey, resulting in what may resemble a semi-structured interview rather than a survey. Consequently, in-person interviews require a lot of personnel and they require proper training to ensure that the survey is conducted according to the protocol, that respondents are treated according to ethical requirements and, that answers are treated in uniform ways.

\footnotetext{
${ }^{4}$ An exception is when questionnaires are prepared in an electronic format (e.g. Questback, Qualtrics) where the researchers may specify that all or certain questions are mandatory, making it impossible to skip a question.
} 
In surveying transitional care across settings, geographical proximity to the research team is an important issue to consider in planning the data collection strategy. If you are surveying stakeholders in a limited geographic area a wider range of approaches may be available compared available survey approaches in a multi-center study. Due to a wide-spread geographic location of the participating centers in the Transitions study, and choosing to administer the questionnaires through in-person interviews, recruiting and training a number of local interviewers in each participating municipality was necessary. The interviewers were mainly geriatric nurses and nursing students trained to administer the questionnaire in an identical manner in every interview. All the interviewers were instructed to ask the exact same questions administered in the same order, following the questionnaire in a highly structured manner. This approach enabled the interviewers to help clarify ambiguous or confusing questions and help the respondents grade their answers by supplying a visual grading scale.

Telephone interviews are an approach that provides a high response rate compared to postal questionnaires and also has the advantage, similar to in-person interviews, of clarifying questions if necessary. In the Transitions study, the interviews with the next of kin were conducted as highly structured telephone interviews. Next of kin such as adult children or other extended family members were also often living in a different geographic area, thus choosing to conduct the interviews on the phone was a logical choice both in terms of resource usage and in acknowledging less need for personal meetings. If we were to conduct the Transition study again today, we might consider using an online questionnaire to survey the next of kin. The tools for conducting online surveys have improved immensely over the last decade, and the internet access and usage has increased in the general population. We believe that the advantages of online surveys may outweigh the disadvantages considering the resource usage in conducting in-person interviews. However, in light of the demographic characteristics of the next of kin in the Transition study, including spouses (often as old and frail as the patients), we fear we would risk excluding the older next of kin from the study if the population was the same. Choosing different modes of data collection in different sub-groups of the population may introduce bias in the data material, which would not be advisable. Consequently, surveys where the population consists of younger age groups online questionnaires may be more appropriate and a logical choice in future studies of transitional care.

\section{Timing of data collection relative to the transition process}

Are there specific assumptions concerning appropriate timing, place, time since transition relative to the type of diagnosis, living conditions etc.? We decided that we wanted to collect data during the 
first period (2-3 weeks) after discharge. The first 2 weeks after a hospital stay is described as an especially vulnerable time. In our study, the respondents were elderly patients who had been sick. The patients needed some time to "settle down" after discharge, and we also wanted to avoid unnecessary burden on the patient by doing the interviews too close to the transition. At the same time, we wanted to collect data closely following the time of discharge to enable secure recollection of the transition process including the hospital stay and after returning to the community. The survey interviews were carried out within two to three weeks after the patient's discharge from the hospital. The telephone interviews with next of kin were conducted within four weeks of the patient's discharge. This time point was chosen because the research team believed this time period to be especially important in gaining valid data on the transition process. The interviewers were able to help the respondents remember details by encouraging their memory by recalling the day things happened by referring to other incidents post discharge. In facilitating interviews early following the hospital discharge the research team was able to gather data on the most immediate experiences of the patients and their next of kin, while they were still in the transition process.

It is possible that the timing of the data collection excluded some patients from consenting to participate, due to an apparent physical frailty in the immediate post-discharge period. Some of the patients did, however, encourage their next of kin to participate as proxy when they themselves felt too frail or were exhausted. The timing of the data collection was a conscious choice to be able to gather data as close as possible to the timeframe in question in the questionnaires. This particular quantitative approach to studying transitional care, using self-report surveys, is more time sensitive than when you study transitional care by examining register data or hospital discharge summaries.

\section{Data analysis}

Surveys conducted by means of structured interviews or questionnaires will generally yield different kinds of numerical data that may be made available for different kinds of statistical analyses. The kinds of analyses that will be available are for the large part dependent on the kinds of data the transitional care questionnaire gathers and the kinds of grading scales used. Thus, the planning stages of the study and the construction of the questionnaire are closely linked to the kinds of data about the transition that the study will yield. Using a cross-sectional survey will not enable the researcher to conclude on cause and effect of a successful transition, although this approach will make it possible to clarify a possible association of for instance discharge planning in relation to successful post discharge outcome. The quality of the research findings will be directly linked to the quality of the design of the study, the quality of the questionnaire, data collection, data entry and 
data analyses. There are a number of challenges to overcome in the process toward dissemination of results and implications to clinical practice.

\section{Preparing the data for statistical analyses}

In research using questionnaires there are several potential challenges in preparing the data file for analysis. In our study we used structured in-person and telephone interviews where the interviewers filled in the questionnaire on paper. The questionnaires were submitted by post to the research team where a research assistant in turn recorded the answers in a SPSS data file following the code book that was developed beforehand. This method of manual data entry is quite vulnerable to human error in all of the stages from interview to data entry, and if possible we recommend using either online data entry to a database or setting up the questionnaires in such a way that it would be possible to scan each questionnaire electronically. Electronic data entry will not eliminate all sources of error, but it may make the process of preparing the file for analyses easier.

In short, preparing the data for analyses entails securing a complete data file without missing data and without invalid scores on individual variables. As mentioned above, securing a complete data file is no small task. There is bound to be some missing data when surveying large study samples. In some cases missing data on some variables may not be a problem, in other cases, for instance in performing analyses with listwise deletion of cases, missing values on any of the included variables excludes that case from all analyses and, thus, there is a risk of decreased sample size and loss of statistical power (Polit and Beck, 2017). There are a number of ways to deal with missing data, we will not go into detail of these procedures in this chapter. However, to illuminate the challenge of missing data we will briefly describe how we dealt with this issue in one of our analyses concerning the patients' self-reported successful post-discharge outcome.

While preparing the SPSS data file for a logistic regression analysis exploring factors predicting a successful post-discharge outcome we uncovered missing values on seven of the variables we intended to include in the analysis. A few missing values on each separate variable is not necessarily cause for concern in a survey, however this was a sub-group analysis of the patients discharged from hospital to home, and we intended to use a statistical analysis method with listwise deletion. Consequently, we needed to impute missing values to the file to make it amenable to analysis. We used the sub-group mean imputation method to impute missing values on the age variable. Missing values on the patient-reported variables measuring ADL-function post-discharge were imputed from the next of kin's answers. This method of imputation gave us variables without 
any missing ADL-values, and we were able to calculate a sum score to be used in the logistic regression. We are aware that patients and next of kin may sometimes disagree regarding their assessment of the patient's functional ability (Knapp and Hewison, 1999). However, we argue that imputing the score reported by the next of kin resulted in a more trustworthy value than imputing the mean score of all the other patients' scores on the variable. The steps we took to secure a complete data file made it possible to perform the statistical analyses that were planned to be able to explore our research questions.

\section{Operationalization of the concept under study}

As mentioned above, one of the main challenges in constructing an instrument to measure a phenomenon lies in successfully operationalizing the concept under study. In our study, one of the main challenges throughout the construction of the questionnaire and through the data analysis was to find a good operationalization of the concept of user participation within the transition process. As we described earlier in the chapter we used the work of Thompson (2007) in operationalizing the concept of participation as a model with three levels of participation:

1. Seeking and/or receiving information - a requirement for participation

2. Engaging in dialogue - the exchange of information

3. Shared decision-making - cooperation between stakeholders

This operationalization made theoretical sense following Thompsons taxonomy, however, we needed to make sure the operationalization also made sense to our sample of respondents in the transition process. To explore this we undertook analyses to ascertain whether or not the questions measuring participation at level 1,2 and 3 did form an ordered scale. In surveys conducted by means of structured interviews or questionnaires, a subset of the survey items with binary (e.g., yes or no) answers forms a Guttman scale if they can be ranked in some order so that, for a rational respondent, the response pattern can be captured by a single index on that ordered scale (Hellevik, 2002). In the case of participation that meant that if a patient or next of kin answered positively to cooperating with the health care personnel (level 3) during the transition process, logically they would also answer positively that they engaged in a dialogue (level 2) and sought or provided information (level 1). In our study, the reproducibility coefficient was $0.85^{5}$ in the patient sample (Foss and Hofoss, 2011), and further analyses showed that the reproducibility coefficient was identical in the responses from the next of kin (Bragstad, 2015). This confirmed that the questions

\footnotetext{
${ }^{5}$ A good Guttman scale should have a coefficient of reproducibility (the percentage of original responses that could be reproduced by knowing the scale scores used to summarize them) above 0.85 .
} 
measuring user participation formed an ordered scale in both populations that we explored. With an $85 \%$ certainty we know that participants who felt they cooperated with the health care personnel also took part in a dialogue and exchanged information during the transition process.

\section{Analyzing and interpreting data concerning post-discharge outcome}

One aim of the Transitions study was to explore factors that could possibly predict a (self-reported) successful post-discharge outcome for the patients (Bragstad et al., 2012). Based on theoretical assumptions derived from earlier research we had reason to believe that factors related to the discharge planning at the hospital, factors related to informal care (provided by next of kin), characteristics of the formal home health care services, and characteristics of the patient might influence the post-discharge outcome. The analysis was controlled for age, gender and length of hospital stay. This is an illustration of how a statistical model based on questions from the DEQ was constructed to explore factors predicting a successful post-discharge outcome - in other words how the DEQ was constructed to measure elements of transitional care. It shows how different questions from a questionnaire can be combined in a multifaceted statistical analysis that enables the researchers to draw conclusions that go beyond the descriptive nature of each separate question. The multiple logistic regression analysis showed that controlled for the other factors in the model, the odds of a successful post-discharge outcome were more than four times higher $(\mathrm{OR}=4.75$, $\mathrm{p}=.022$ ) for patients reporting that someone was present when they came home than for those who came home to an empty house. Patients reporting that they thought the help they received from the municipality was adequate had an odds four times (OR 4.18, $\mathrm{p}=.006)$ higher of reporting a successful outcome than those who thought the help was inadequate. The patients' age, gender, length of stay, ADL and IADL function, whether they received help from family and friends, lived alone, reported being surprised by the timing of the discharge or whether they reported that there was a discharge planning conference were not statistically significant predictors in this model (Bragstad et al., 2012). The questionnaire data from the DEQ in the Transitions study, enabled the research team to interpret the findings in line with earlier research which has pointed towards the inadequacy of municipal home-care services and, furthermore, confirm that informal help from family and friends is an important supplement to the formal home care services provided by the municipality. In our logistic regression model ADL and IADL function were not statistically significant with regard to the post-discharge outcome. That is not to say that the patient's functional status does not affect the post-discharge outcome, it probably just means that the patient's functional dependency was compensated for by the amount of formal and informal help received post-discharge. 


\section{Generalizability of the findings}

It is always important to consider the representativeness of the sample recruited to participate in a survey, is the sample representative of the target population? The patients who participated in the Transitions study were not representative of all patients transitioning from hospital to community care; they were a selected group of patients aged 80 years and older. The sample characteristics were carefully compared to the general population aged 80 years and older, and our analyses showed that the sample did indeed represent the population in gender distribution, level of education and marital status. This knowledge enables us to trust that our findings regarding patient participation represents that of the general population.

Pertaining to the generalizability of findings from a survey it is important to be aware that all decisions made during construction of the questionnaire, issues concerning data collection and analyses may all influence the results. The way we conceptualize transitional care and how we explore the concept will affect the results we produce. In essence, the questions we ask in the questionnaire control the responses we receive and consequently also the results we disseminate.

\section{Conclusion}

In this chapter we have drawn attention to some challenges in the design and execution of a survey on transitional care. We have done so by focusing on some methodological issues related to the different phases of a survey. Transitional care is, as mentioned a complex field of research. Transition can be between or within levels of care and organizations and involve a range of different professions. Patients involved are in different situations, of different ages, gender, social situations, vulnerabilities etc. Next of kin may be close or distant to us or to the patient. All this is our reason for highlighting the need for specification at all levels of the research process. It is also a reason for advocating research that uses different sources of data, enabling us to compare the views on transition processes for instance between nurses in primary care versus nurses in hospital care or between nurses and patients. To enable such a comparison it is necessary to develop instruments that make comparison possible by asking comparable questions. We consider the issues that we have chosen to highlight as central, but unequivocally acknowledge that there are other issues that are also important. Reflexivity is vital throughout all stages of the project. We end this chapter by summarizing some key recommendations drawn from our work in the Transitions study (Table 1). 
Table 1. Key recommendations

\begin{tabular}{ll}
\hline Phase & Key recommendations \\
\hline $\begin{array}{l}\text { Designing and } \\
\text { planning the } \\
\text { study }\end{array}$ & $\begin{array}{l}\text { Specify the purpose of the study } \\
\text { Carefully consider the population you want to survey } \\
\text { Choose appropriate time (relative to transition process) for conducting } \\
\text { survey }\end{array}$ \\
Development of & Operationalization of concepts of study \\
the instruments & Careful consideration of content of questionnaire based on knowledge of \\
& the transitional care process \\
Carefully construct unambiguous questions and response alternatives \\
Conduct a pilot study to test the questionnaire and ascertain feasible sample \\
selection and recruitment \\
Establish close collaboration with the personnel recruiting study \\
$\begin{array}{l}\text { on transitional } \\
\text { care }\end{array}$ & $\begin{array}{l}\text { Consider available and necessary resources to conduct the survey } \\
\text { Implement chosen data collection mode } \\
\text { Allocate appropriate resources to monitor data collection } \\
\text { Allow for time to prepare data for analysis } \\
\text { Critically appraise the operationalization of the concept(s) under study } \\
\text { Adhere to statistical analysis plan } \\
\text { Evaluate generalizability of the findings }\end{array}$ \\
\hline
\end{tabular}




\section{References}

ABMA, T. A., NIERSE, C. J. \& WIDDERSHOVEN, G. A. M. 2009. Patients as Partners in Responsive Research: Methodological Notions for Collaborations in Mixed Research Teams.

BRAGSTAD, L. K. 2015. Indispensable intermediaries : the role of informal caregivers in the discharge process of older relatives. no. 1959, Institute of Health and Society, Faculty of Medicine, University of Oslo.

BRAGSTAD, L. K., KIRKEVOLD, M., HOFOSS, D. \& FOSS, C. 2012. Factors predicting a successful post-discharge outcome for individuals aged 80 years and over. International Journal of Integrated Care, 12, e147.

BRAGSTAD, L. K., KIRKEVOLD, M., HOFOSS, D. \& FOSS, C. 2014. Informal caregivers' participation when older adults in Norway are discharged from the hospital. Health \& social care in the community, 22, 155-168.

COLEMAN, E. A. 2003. Falling through the cracks: challenges and opportunities for improving transitional care for persons with continuous complex care needs. Journal of the American Geriatrics Society, 51, 549-555.

FOSS, C. 2011. Elders and patient participation revisited - a discourse analytic approach to older persons' reflections on patient participation. Journal of clinical nursing, 20, 2014-22.

FOSS, C. \& ASKAUTRUD, M. 2010. Measuring the participation of elderly patients in the discharge process from hospital: a critical review of existing instruments. Scandinavian Journal of Caring Sciences, 24, 46-55.

FOSS, C. \& HOFOSS, D. 2011. Elderly persons' experiences of participation in hospital discharge process. Patient Educ Couns, 85, 68-73.

HELLEVIK, O. 2002. Forskningsmetode $i$ sosiologi og statsvitenskap [Research methods in sociology and political science], Oslo, Universitetsforlaget.

KEARNEY, M. H. 2016a. Under - Appreciated Steps in Instrument Development, Part I: Starting With Validity. Research in Nursing \& Health, 39, 81-83.

KEARNEY, M. H. 2016b. Under - Appreciated Steps in Instrument Development, Part II: Ending With Validity. Research in Nursing \& Health, 39, 135-138.

KNAPP, P. \& HEWISON, J. 1999. Disagreement in patient and carer assessment of functional abilities after stroke. Stroke, 30, 934-8.

POLIT, D. F. \& BECK, C. T. 2017. Nursing Research : generating and assessing evidence for nursing practice, Philadelphia, Wolters Kluwer.

THOMPSON, A. G. 2007. The meaning of patient involvement and participation in health care consultations: a taxonomy. Social science \& medicine, 64, 1297-310. 